\title{
Cathodoluminescence and depth profiles of tin in float glass
}

\author{
B Yangt, P D Townsend and S A Holgate \\ MAPS, University of Sussex, Brighton BN1 9QH, UK
}

Received 9 March 1994

\begin{abstract}
Cathodoluminescence (cL) spectra have been recorded from float glass samples and the data used to determine the depth profile of tin introduced from the float process. The luminescence data were interpreted in terms of intrinsic emission bands at 380 and $410 \mathrm{~nm}$ and well resolved extrinsic emission bands resulting from the tin impurities at 575 and $630 \mathrm{~nm}$. Signals were recorded at temperatures from 40 to $300 \mathrm{~K}$. The presence of the tin produces an optical waveguide in the glass surface as a result of the float production process. It was therefore possible to depth profile the tin by measurement of the waveguide modes. The $c L$ and waveguide methods are in excellent agreement. Overall, the depth of tin diffusion was up to $8-9 \mu \mathrm{m}$ in the samples studied. The lifetimes and intensity of the signals are temperature dependent, and these parameters are reported for the $380 \mathrm{~nm}$ and $575 \mathrm{~nm}$ emission lines. Thermal treatments modify the $\mathrm{CL}$ spectra and such changes are considered the result of valence or aggregation states of the tin impurities.
\end{abstract}

\section{Introduction}

Whilst float glass is a familiar material for building applications, it should be recognized that there are some interesting properties which have not been exploited or studied in detail for other applications. Foremost among these is that the float process inevitably introduces tin, by thermal diffusion, into one face of the glass [1]. The presence of the tin raises the refractive index and consequently forms a very low-loss planar waveguide $[2,3]$. Applications for such large area planar waveguides have not yet been publicly proposed. At a more fundamental level it is worth enquiring if the concentration profile of the tin and the refractive index changes are the same. Further, one should consider if the tin is in the optimum valence state for such changes in refractive index, and whether one can control the state of incorporation of tin within the glass. The present paper addresses these questions using the two totally independent techniques of waveguide mode profiling and cathodoluminescence spectral measurements.

\section{Experimental details}

Measurements were made on float glass samples from both Societa Italiana Vetro (SIV) and from Pilkington plc. Since the luminescence signals were very similar, only the data from SIV glass are reported here. The samples were cut into $10 \times 10 \mathrm{~mm}^{2}$ pieces and cleaned

$\dagger$ Permanent address: Physics Department, Beijing Normal University, Beijing 100875, China before examination. The thickness of these samples was $3 \mathrm{~mm}$. For the cathodoluminescence, an electron beam of energy $10 \mathrm{keV}$ was focused onto a spot of approximately $2 \mathrm{~mm}$ in diameter. Beam currents of $0.03 \mu \mathrm{A}$ were used to excite the samples. Since changes in beam energy and current density might modify the emission spectra and intensity, for the present paper all the spectra shown here were recorded with the same beam conditions. The electron beam was chopped at a frequency of $90 \mathrm{~Hz}$, except for the lifetime measurements, and the signal output was measured with a lock-in amplifier.

Spectra were measured in the temperature range 40 to $300 \mathrm{~K}$ using an $f / 4$ scanning monochromator and a cooled red-sensitive photomultiplier tube. All data were recorded on a computer and corrected for the spectral response of the whole detection system, as described earlier [4]. A spectral resolution of $10 \mathrm{~nm}$ was used.

Depth profiles using the optical waveguide properties of the tin side of the glass were made by measuring the waveguide mode spacing with both a $632.8 \mathrm{~nm}$ HeNe laser and a $488 \mathrm{~nm}$ argon beam by the standard dark mode prism coupling method [5]. The depth profile was then determined using a reflectivity analysis described by Lama and Chandler [6].

\section{Results}

\subsection{Optical waveguide data and depth analysis}

The float process produces flat glass by allowing the liquid glass to spread across a sheet of molten 


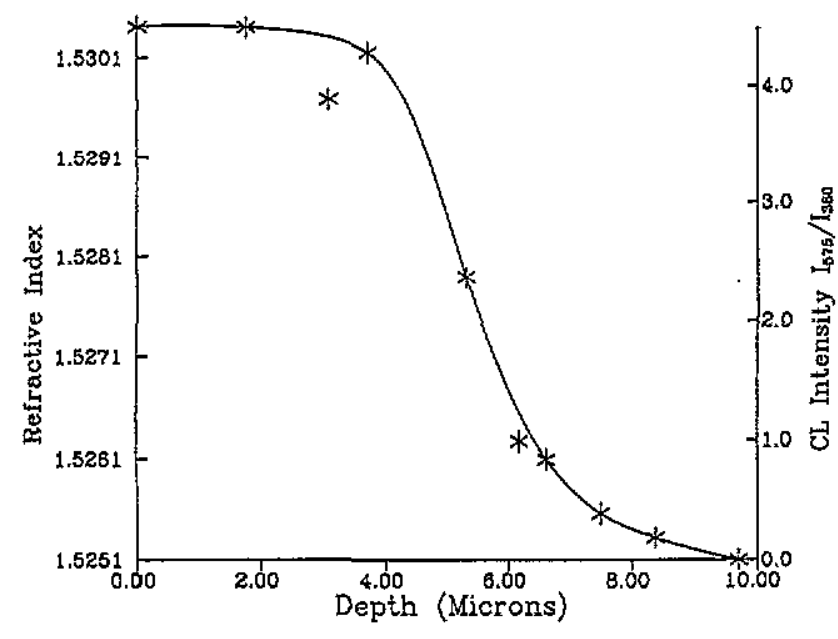

Figure f. A comparison of the refractive index and cathodoluminescence changes produced by tin in a float glass sample. The full curve is the index profile determined from optical mode analysis. Cathodoluminescence data are shown after several polishing steps.

tin. During this high-temperature phase of the glass production tin diffuses into the lower face of the glass to a depth of several micrometres. There is also some tin contamination of the upper face of the glass from the tin vapour, although this is in much smaller concentrations. The upper face tin content is sensitive to the furnace conditions and so varies not only with the supplier but also with the glass thickness and flow rate across the tin bath. The two faces are readily distinguishable since the tin-rich side fluoresces when exposed to UV illumination. Less obviously, one may detect the change in refractive index and surface reflectivity resulting from the tin impurities. Since the tin raises the refractive index of the glass, the layer is potentially an optical waveguide $[2,3]$. Indeed such glass layers provide extremely low cost, very large planar waveguides of excellent quality and low optical loss [2]. To a first approximation one may assume that the refractive index enhancement within the waveguide is proportional to the concentration of the tin impurity ions. In order to obtain the diffusion profile of tin in the surface of the glass, the following method has been used. The conventional waveguide profiling method is to couple a laser beam into the waveguide via a prism. Measurement of the critical angles at which the mode indices within the guide match the coupling reveal the mode spacings within a multimode guide. Such data were obtained with both a red HeNe laser at $632.8 \mathrm{~nm}$ and a blue argon line at $488 \mathrm{~nm}$. The profile shapes were determined by waveguide mode analysis, although there are only a limited number of measurable modes, with a maximum of four modes, even for the shorter wavelength. Nevertheless, one can estimate the refractive index profile from the mode spacing, and compare the profiles from the two sets of wavelength data. In practice these were in excellent agreement. They lead to the profile of the form shown by the continuous line in figure 1 . The profile shows that there is initially a saturation value, to a depth of $\approx 2.95 \mu \mathrm{m}$. This is followed by a tail in the refractive index profile which is of a Gaussian shape, as expected for a diffusion process. Overall the tin penetration extends to beyond $8 \mu \mathrm{m}$ for the example chosen.

Since the mode spacings are dependent on the index profile, and thus the depth of the waveguide, the mode values may be used to monitor the effects of sectioning of the guide as surface layers are successively removed by polishing. Using fine diamond power of $1 \mu \mathrm{m}$ diameter, the Sn surface of the sample was polished, step by step, so as to remove the tin diffused layer. As the tin diffused glass is a planar waveguide the movement of the mode indices are a function of guide thickness, and so determine the extent of the residual tin layer. The multimode measurements was adequate so long as the layer was thicker than $5 \mu \mathrm{m}$. After some practice, a linear relationship between the thickness polished from the layer and the polishing time was established. The polishing rate was constant with time, and so it was possible to predict the thickness of the residual doped layer even when the layer was thinner than $5 \mu \mathrm{m}$. (This information on polishing rate was required for the parallel experiment using cathodoluminescence.) The refractive index profiles determined after each polishing step were in excellent agreement, and the profile curves for the various residual thicknesses defined the same form for the index changes for the inner part of the diffused glass.

\subsection{Cathodoluminescence data}

The cathodoluminescence emission spectrum and intensity are not easily interpreted in the case of a nonuniform dopant situation, since the rate of energy deposition from the electron beam, is a function of depth and, at least in principle, the efficiency of light production and the spectrum can be sensitive to the rate of excitation. There are therefore several problems to address in using $\mathrm{CL}$ to monitor the presence of impurities in a quantitative fashion. The first is to ascertain if the spectrum has a signal which is uniquely linked to the impurity, the second is to assume that the signal is linearly dependent on the dopant concentration in the glass, and insensitive to the rate of electron beam energy deposition. For float glass the $\mathrm{Sn}$ impurity is clearly dominant on the side which was in contact with the tin. Nevertheless, some tin reached the upper face by vapour transport. However, tin is unlikely to reach the interior. Hence some of the SIV glass was cut to provide thinner pieces of $10 \times 10 \times 1.5 \mathrm{~mm}^{3}$. The objective of such a cutting operation was to provide three distinct types of surface. One, the face labelled Sn corresponds to the side of the glass in contact with the molten tin during the float production. The opposite (i.e. the non-tin) side, labelled $\mathrm{N}$, has become lightly doped with tin vapour. The inner faces, labelled $I$, exposed by cutting the sample, are assumed to be essentially free of tin.

The CL spectra of these three surfaces are distinctly different and are shown in figure 2. The following features can be deducted from this figure. 
(1) Two peaks at 380 and $410 \mathrm{~nm}$ always exist in the luminescence from interior sections of the glass, and so are almost certainly the result of intrinsic emission processes. These intrinsic emission bands have a long wavelength tail extending over all the visible range. The intensity of the tail decreases with longer wavelengths and contains several component bands, as discussed in a later section.

(2) The strong peak at $575 \mathrm{~nm}$ and the broad shoulder peaked near $630 \mathrm{~nm}$ undoubtedly result from tin emission bands, because these bands are extremely strong in the spectrum of the $\mathrm{Sn}$ surface, and do not appear in that of the I surface. Two types of additional measurements confirm this. The first is that the intensity of the signals from the so called non-tin side is approximately linear with glass thickness and this is closely related to the time spent in the float section of the furnace. Data from glass coated with a deposit of $\mathrm{SnO}_{2}$ produced broadly the same strong features. The $575 \mathrm{~nm}$ peak was still dominant but some of the weaker features differed slightly from that of the float glass. In particular the $630 \mathrm{~nm}$ broad band in the float was split into two components near 610 and $645 \mathrm{~nm}$ and the shorter wavelength peaks were better resolved. Overall these results confirm the role of the tin but suggest that a number of emission bands may be hidden with the envelope described here.

(3) Weaker components on the short wavelength side of the $\mathrm{Sn}$ band (e.g. near 460 and $515 \mathrm{~nm}$ ) are noted but it is difficult to decide whether they relate directly to the tin, intrinsic sites, or other luminescence sites formed for example as intrinsic/tin complexes. In the data for the non-tin side versus glass thickness the intensity of these bands relative to the $575 \mathrm{~nm}$ Sn signal varied in the same way as did that for the intrinsic $380 \mathrm{~nm}$ signal. This suggests that these smaller features are not primarily from transitions at tin sites.

(4) As mentioned in (2), in the CL spectrum of the upper $(\mathrm{N})$ surface, the tin bands were also detected, although their intensities are much lower than those from the face in contact with the tin. They were also variable in intensity and the ratio of the component bands was not constant in all samples. It can be estimated that the tin concentration in the $\mathrm{N}$ surface is about $16 \%$ of that in the Sn surface for the $3 \mathrm{~mm}$ thick samples (assuming a linear intensity relationship between the CL signal and the tin concentration). The intensity of the tin signal from the upper face is approximately proportional to the time spent during the passage of the glass over the tin zone.

\subsection{Cathodoluminescence and depth analysis}

On the float side of the glass, with a high tin concentration, it is possible to compare the refractive index profile with the $\mathrm{Cl}$ intensity. In order to avoid secondary effects from CL depth profiling using variable electron energy it is preferable to use fixed electron beam conditions, and selectively remove material from the glass surface by polishing. One notes that the electron

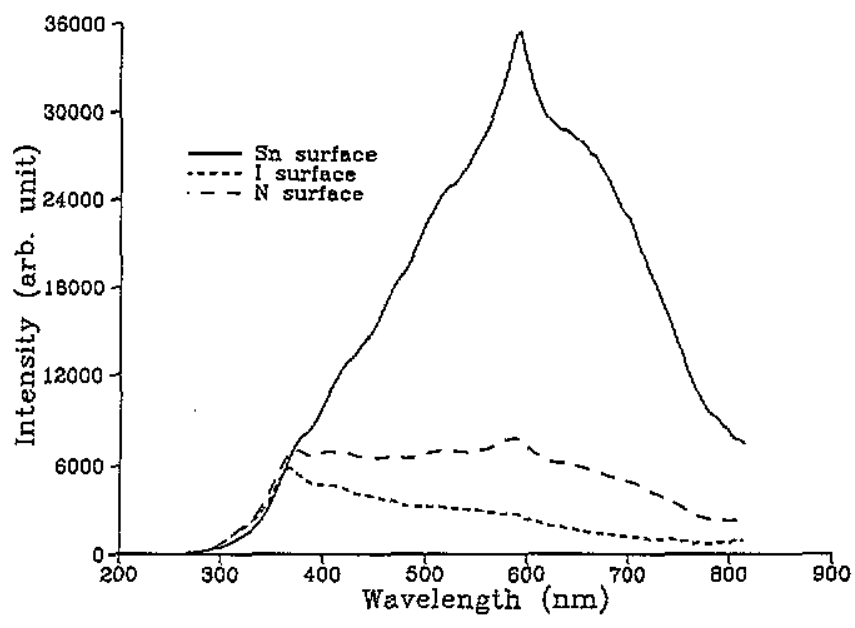

Figure 2. A comparison of the $\mathrm{CL}$ spectra from the tin side (Sn), the non-tin side (N) and the interior (I) of float glass.

probe beam has an energy against depth excitation profile which is very approximately that of a Gaussian line shape, and the maximum electron range of $10 \mathrm{keV}$ electrons in glass is near half a micrometre. However, since the glass surface is angled relative to the ion beam in order to record the emission spectra, the effective $\mathrm{CL}$ analysis is only from a depth of some $0.3 \mu \mathrm{m}$. Deconvolution of the electron energy deposition profile and the depth dependence of the signal on a finer depth scale was thus not attempted. For the fixed beam condition the CL spectrum was recorded after every polishing step, and compared with the waveguide analysis. CL experimental conditions are difficult to reproduce precisely and so a signal normalization was used in which the $575 \mathrm{~nm}$ emission, from tin, was compared with that near $380 \mathrm{~nm}$, which is considered to be an intrinsic signal of the glass host. The $575 \mathrm{~nm}$ luminescence signal is assumed to be proportional to the tin concentration and further, one assumes that the presence of tin does not influence the efficiency of light production at $380 \mathrm{~nm}$. During profiling the intensity data of the $575 \mathrm{~nm}$ emission relative to that at $380 \mathrm{~nm}$ are superposed on the waveguide data of figure 1 . The form of the index and CL profiles are in good agreement. The linearity of the polishing rate allows the $\mathrm{CL}$ analysis to be extended into the tail of the index profile, even beyond the point when mode values offered depth information. The important point to note is that the two totally distinct approaches to the tin profiling offer the same depth penetration profiles for the tin.

\subsection{Thermal treatments}

During glass production the material cools whilst in the form of a massive sheet of glass. This cooling phase can influence the manner in which the tin is incorporated into the glass network. Alternative possibilities include different degrees of coalescence of the tin, for example as colloidal precipitates, or as tin in different charge states [7]. Colloidal metal precipitation is probably unimportant, since there was very low loss and scattering in the earlier waveguide loss data [2]. However, the 


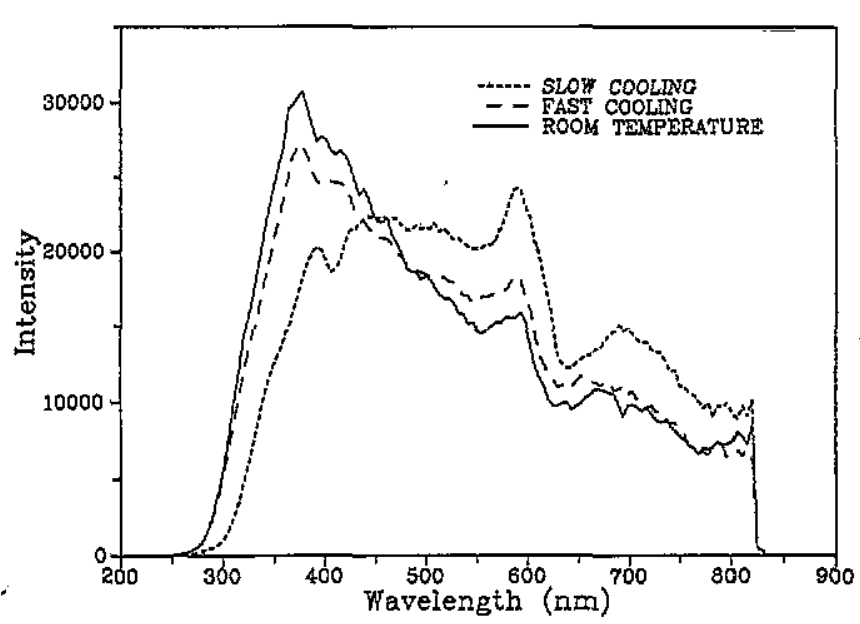

Figure 3. Changes induced in the $\mathrm{CL}$ spectra from the non-tin side after heating to $600^{\circ} \mathrm{C}$ and either cooling quickly or slowly.

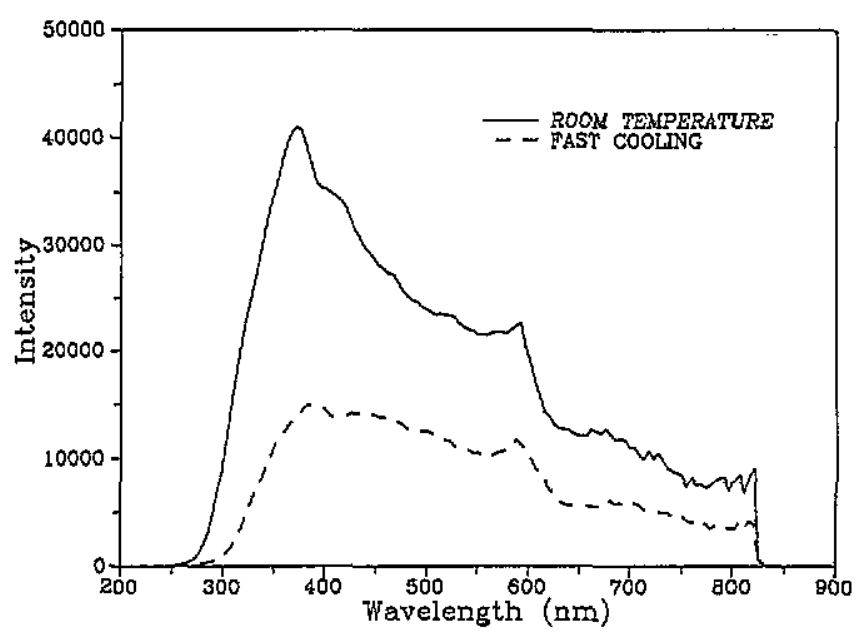

Figure 4. An example of $\mathrm{cl}$ spectra from an interior glass surface before and after fast cooling from $600^{\circ} \mathrm{C}$.

identification of alternative valence states of the tin is a more difficult problem. Assuming that changes in valence might be reflected by variations in intensity or luminescence spectra, an attempt was made to modify the spectra by thermal treatments. With this objective in mind the glass samples were heated to various temperatures up to $600^{\circ} \mathrm{C}$, and then either slowly cooled (down to $300^{\circ} \mathrm{C}$ in about $5 \mathrm{~min}$ ) or rapidly removed from the furnace and placed on a cold metal surface. In principle this could establish and stabilize different degrees of imperfection, or valence, for impurities or intrinsic defects in the glass. In practice the CL data from the tin side were insensitive to the various treatments, but both the non-tin and interior faces gave signals which were obviously modified. The pattern of changes is complex, as it depends on both the maximum furnace temperature used and the cooling rate. In order to demonstrate the scale of the changes, examples are given in figure 3 for a 'fast' and 'slow' cooled glass recorded from the non-tin side. Figure 4 shows similar data for interior faces of the glass. The maximum temperature used for these examples was $600^{\circ} \mathrm{C}$.

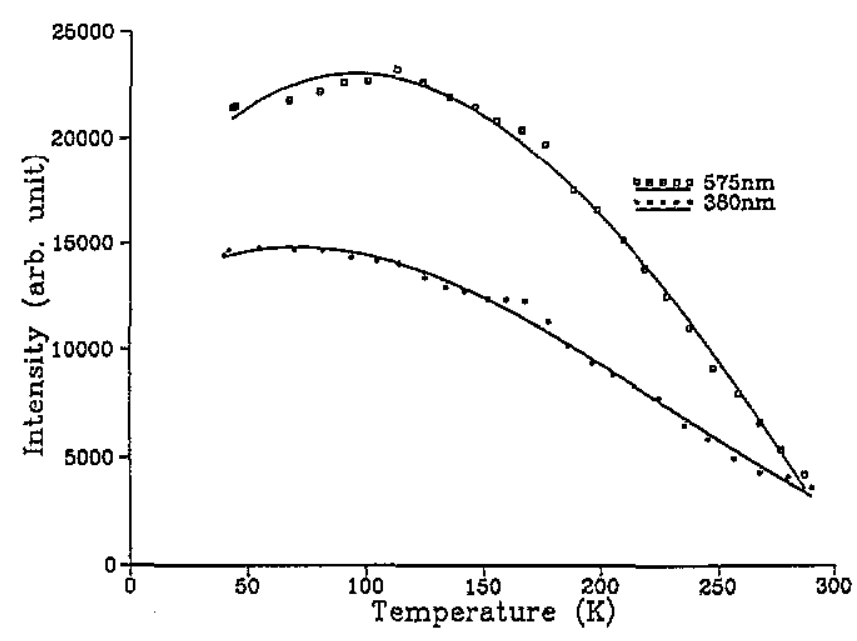

Figure 5. $\mathrm{cL}$ intensity of the $575 \mathrm{~nm}$ and $380 \mathrm{~nm}$ bands of float glass as a function of temperature.

\subsection{Lifetime and temperature dependence}

Intensities of the $380 \mathrm{~nm}$ and $575 \mathrm{~nm} \mathrm{CL}$ emission bands of the Sn surface of SIV glass were recorded at various temperatures from 300 down to $40 \mathrm{~K}$ and the results are shown in figure 5 . One may characterize such temperature variations by an activation energy $E$, by assuming that there is a temperature insensitive component plus a thermally activated element to the signal. Effectively this assumes that there is a thermally activated balance between the decay routes for radiative and non-radiative emission. This evaluation is based on the fact that in the higher temperature range the additional emission intensity $\Delta I$ is proportional to $\exp (-E / k T)$, where $\Delta I$ is equal to $\left(I_{0}-I\right)$, and $I_{0}$ and $I$ are the maximum intensity and the intensity at temperature $T$ of the same emission band respectively. The relationships between $\ln (\Delta I)$ and $1 / T$ of the $380 \mathrm{~nm}$ and $575 \mathrm{~nm}$ bands are linear over the temperature range 267 to $188 \mathrm{~K}$. The gradients of the linear regions correspond to values of $E$ of $0.046 \mathrm{eV}$ and $0.064 \mathrm{eV}$ for the $380 \mathrm{~nm}$ band and $575 \mathrm{~nm}$ bands respectively.

Additionally, the phase difference $\phi$ between the reference and signal can be measured by using the lockin amplifier. Hence, in principle, the signal lifetime $\tau$ can be obtained from the relation $\tan \phi=2 \pi v \tau$, where $v$ is the chopping frequency. Unfortunately in the present $\mathrm{CL}$ system the maximum chopping frequency is only $100 \mathrm{kHz}$ and this is slow compared with the luminescence lifetimes being considered. Nevertheless, attempts to estimate the lifetime of the $380 \mathrm{~nm}$ intrinsic emission gives a value near $0.8 \times 10^{-6} \mathrm{~s}$ at room temperature (RT), and near $1.0 \times 10^{-6} \mathrm{~s}$ at $40 \mathrm{~K}$. The values for the $575 \mathrm{~nm}$ tin band are probably longer, with values near $1.1 \times 10^{-6} \mathrm{~s}$ at RT, and $1.2 \times 10^{-6} \mathrm{~s}$ at $40 \mathrm{~K}$. As the lifetimes of all the emission bands are similar to each other, and too short for the present CL system, it was not possible to improve the resolution of the overlapping bands by using different chopping frequencies. 


\section{Discussion}

The immediately obvious feature from figure 1 is that the independent monitors of the effects of tin diffusion, namely via the refractive index and the longwavelength $\mathrm{CL}$ provide the same depth profile. One can thus attribute such changes to the penetration of tin into the glass. The data suggest that there is a saturation region near the surface followed by a Gaussian-type diffusion tail extending down to some $8 \mu \mathrm{m}$ depth. Intuitively this is a reasonable consequence of the float process. However one should note that the analyses are on a relatively coarse depth scale and thus smaller scale variations are smoothed over. Earlier analyses of the depth concentration of the $\mathrm{Sn}$ [7-11] using SIMS, RBS, Auger and Mossbauer techniques are not totally consistent. They generally show an overall pattern of a decreasing concentration with depth, but in some cases there are indications of a peak in the concentration close to the surface (at depths of, say, $100 \mathrm{~nm}$ ). Such subtleties are not resolvable by the current techniques.

In order to analyse the luminescence data one should note that the SIV glass contains more than $70 \%$ silica, and some alkali and alkaline earth oxides, e.g. $\mathrm{Na}_{2} \mathrm{O}, \mathrm{CaO}$ and $\mathrm{MgO}$. For such a glass the atomic structure has a short-range regular arrangement of atoms but a complete lack of any long-range periodicity and regularity. The silica tetrahedron $\left(\mathrm{SiO}_{4}\right)^{4-}$ block is the most important molecular building unit and the glass lattice network is formed from such units, but the oxygen ions may also be linked to an alkali metal or an alkaline-earth metal ion. The intrinsic emission from the silicate group has been studied in many types of sample, including such diverse examples as silica and quartz, silica fibres, feldspars and zircon (e.g. see reviews in [4,12-14]). Despite the wide selection of host structures there are many similarities with a general pattern of blue/UV emission in the wavelength range 300 to $480 \mathrm{~nm}$, which is variously ascribed to intrinsic luminescence emitted from relaxed self-trapped excitons decaying at the intrinsic $\left(\mathrm{SiO}_{4}\right)$ site, or wavelength shifted variations of this recombination resulting from the presence of defects such as the intrinsic examples of $E^{\prime}$ or $E_{\beta}$ centres, or from common impurities such as $\mathrm{H}, \mathrm{Al}$ or $\mathrm{Ge}$. Consequently, for the present data, the assumption is that the emission bands near $380 \mathrm{~nm}$ and $410 \mathrm{~nm}$ in the blue end of the spectrum of this silicate glass are similarly related to intrinsic electron hole recombination. In some of the samples the $380 \mathrm{~nm}$ signal was more pronounced after heat treatment. Since there are considerable variations in the assignments of the origin of the defects which produce luminescence, even at the same wavelength, table 1 lists some of the more common interpretations.

$\mathrm{CL}$ is not a passive probe of the defect sites and, whilst changes in energy obviously sample different depths of material, variations in current at the same electron beam energy may alter the relative intensities of different emission bands. In part this is because for an insulator the secondary electron emission and space charge development induce a complex electric
Table 1. Examples of emission bands seen in silicate materials and tentative assignments of their origin.

\begin{tabular}{llll}
\hline \multicolumn{2}{l}{ Band position } & & \\
\cline { 1 - 2 }$(\mathrm{nm})$ & (eV) & Method & Possible model \\
\hline 260 & 4.77 & $\mathrm{CL}$ & Oxygen vacancy \\
290 & 4.28 & $\mathrm{PL}, \mathrm{CL}, \mathrm{TL}$ & Oxygen vacancy \\
370 & 3.78 & $\mathrm{RL}, \mathrm{TL}$, & $\mathrm{H}_{3} \mathrm{O}_{4}$ \\
380 & 3.25 & $\mathrm{RL}, \mathrm{CL}, \mathrm{TL}$ & Seen in ultrapure quartz \\
390 & 3.17 & $\mathrm{FL}$ & Oxygen vacancy \\
400 & 3.1 & $\mathrm{PL}, \mathrm{TL}$ & $\mathrm{O}_{2}$ intrinsic/Ge impurity \\
$450-480$ & $2.5-2.8$ & $\mathrm{FL}, \mathrm{CL}$ & Self-trapped exciton \\
470 & 2.64 & $\mathrm{PL}, \mathrm{TL}$ & AlO \\
570 & 2.18 & $\mathrm{CL}$ & Ge impurity \\
580 & 2.15 & $\mathrm{CL}$ & Oxygen vacancy \\
640 & 1.9 & $\mathrm{CL}, \mathrm{IB}$ & Na impurity \\
\hline
\end{tabular}

Note: 'Oxygen vacancy' covers alternative versions of the structural defects such as $E^{\prime}, E_{\beta}^{\prime}$, etc. These bands have been reported for at least those methods shown based on recent literature. CL, PL, RL, TL are cathodo-, photo-, radio- and thermoluminescence respectively. IB is ion beam stimulated.

field distribution in an insulating glass [15]. The scale of the changes in surface potential may be comparable to the primary electron beam energy. Surface states can modify the alternative radiative and non-radiative decay routes of the excited material and hence alter the intensities of different emission bands. As evidence for such effects, figure 6 shows the CL intensity of $380 \mathrm{~nm}$ and $575 \mathrm{~nm}$ bands as a function of the electron beam current. Note that since both signals are recorded together from the same sample the differences in $\mathrm{CL}$ intensity are not simply experimental artefacts. One notes that the relationship between the intensity of the $380 \mathrm{~nm}$ emission and the beam current is slightly super-linear. Numerous factors might play a role in these similar, but differing, responses of the two main emission bands confirm that they are of distinctly different origin. For example, we may speculate that the 380 and $575 \mathrm{~nm}$ emissions are maximized at different depths within the electron penetration range, and so are variously influenced by surface charging and internal electric fields. Such factors would result in a changed sensitivity to current density or local beam heating. Such a suggestion is prompted by the knowledge that the surface composition of glass frequently differs from that of the interior, particularly as a result of alkali ion migration and surface penetration of water.

When the tin atom, with the electronic configuration ${ }^{5} \mathrm{~S}_{2}$, ${ }^{5} \mathrm{P}_{2}$, diffuses into the glass, electron transfer can affect one or more electrons. So the tin may be incorporated in the glass as an interstitial atom, or as Sn linked with either one or two oxygen ions. Earlier studies using Mossbauer spectroscopy indicate that the ratio of $\mathrm{Sn}^{+2}$ to $\mathrm{Sn}^{+4}$ states is a function of depth and production conditions [7]. One assumes that each type of ion site can produce characteristic emission features which, in principle, may be associated with the observed tin emission bands observed in the present work. The heat treatments modified the spectra on the non-tin side 


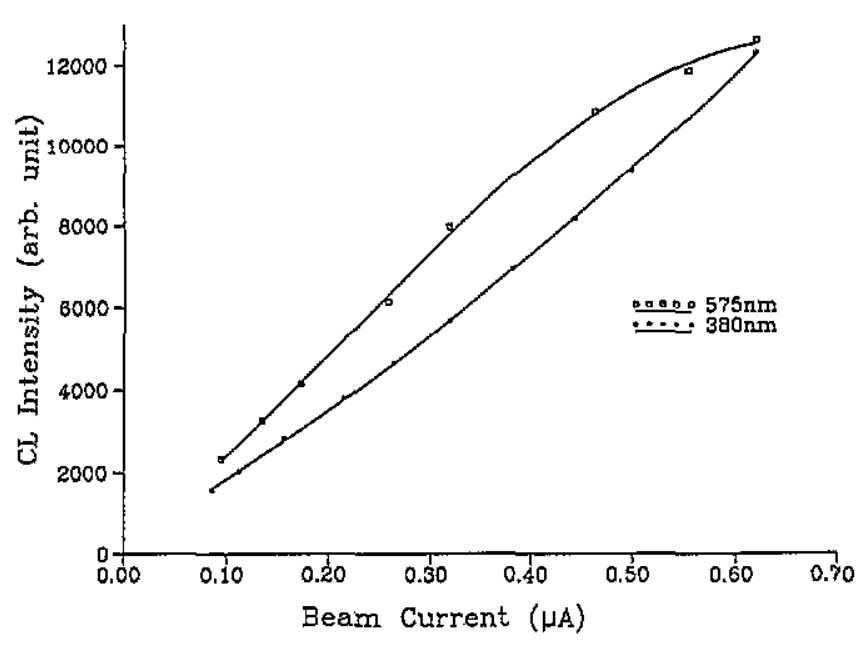

Figure 6. CL intensity of the $575 \mathrm{~nm}$ and $380 \mathrm{~nm}$ emission bands of float glass against electron beam current at room temperature.

of the glass but on the float side the changes were minimal, implying that at the high tin dopant levels the glass had reached a saturation equilibrium. This view is confirmed by the refractive index data where the index is constant for nearly $3 \mu \mathrm{m}$ into the glass.

\section{Conclusion}

In conclusion one notes that the introduction of tin into float glass directly influences the refractive index and the CL intensity. Emission bands near 575 and $630 \mathrm{~nm}$ are associated with the tin. The float process leads to a saturation of the signals near the glass surface which was in contact with the molten tin. One assumes that tin diffuses into the glass in the molten phase and the fact that both the index and CL signals show a surface saturation, followed by a Gaussian tail deeper in the glass, suggests that there is a linear correlation between the tin concentration and the observed changes. The opposite glass face, doped by vapour transport is not in equilibrium and subsequent thermal processing can alter the state of the tin within the glass network.

\section{Acknowledgments}

We are grateful to the Royal Society for financial support. The work is also part of the EEC BRITE. EURAM programme, contract BREU-0357. We further wish to thank Societa Italiana Vetro for encouragement and helpful discussions.

\section{References}

[1] Pilkington L 1969 Proc. Roy. Soc. A 3141

[2] Kakarantzas G, Glavas E and Townsend P D 1989 Electron. Lett. 25102

[3] Gevorgyan S Sh, Zabunyan $\mathrm{MH}$ and Vrtanessyan $\mathrm{G} \mathrm{S}$ 1989 Opt. Quant. Electron, 21307

[4] Luff B J and Townsend P D $1990 \mathrm{~J}$. Phys.: Condens. Matter 28089

[5] Tien P K, Ulrich $R$ and Martin R J 1970 J. Opt. Soc. Am. 601325

[6] Lama F L and Chandler P J 1986 Opt. Acta. 33127

[7] Principi G, Maddalena A, Gupta A, Geotti-Bianchini F, Hreglich S and Verita M 1993 Nucl. Instrum. Methods B 76215

[8] Colombin L, Jelli A, Riga J, Pireaux J J and Verbist J 1977 J. Non-Cryst. Solids 24253

[9] Colombin L, Charlier H, Jelli A, Debras $G$ and Verbist J 1980 J. Non-Cryst. Solids 38/39 749

[10] Baitinger W E, French P W and Swarts E L 1980 J. Non-Cryst. Solids $38 / 39749$

[11] Verita $M$, Geotti-Bianchini $F$, Hreglich $S$, Pantano S G and Bojan V 1992 Bol. Soc. Espana. Ceram. Vid. 31-C 415

[12] Yang X H and McKeever S W S 1988 Nucl. Tracks Radiat. Meas. 14 75; 1990 J. Phys. D: Appl. Phys.23 237; 1990 Radiat. Prot. Dosim. 3327

[13] Song K S and Williams R T 1993 Self Trapped Excitons (Berlin: Springer)

[14] Khanlary M R, Townsend P D and Townsend J E 1993 J. Phys. D: Appl. Phys. 26371

[15] Cazaux J 1986 J. Appl. Phys. 591418 